\title{
Covid-19 Era Healthcare-Worker Suicide Prevention
}

Lai Fong Chan

Prof. Chan Lai Fong is currently Associate Professor of Psychiatry \& Consultant Psychiatrist at the National University of Malaysia. She trained in psychiatry at the National University of Malaysia and completed a Clinical Fellowship in Mood \& Anxiety Disorders at the University of Toronto, followed by a Master of Science in Affective Neuroscience at Maastricht University. Prof. Chan's research interests are in the area of gene-environment, as well as socio-cultural interactions in suicidal behavior among adolescents, young adults and high-risk clinical populations i.e. treatment-resistant depression, bipolar disorder \& SLE. She was recently awarded the IASP 2017 De Leo Fund Award for Outstanding Research on Suicidal Behaviours in Developing Countries.

Abstract. As front-liners bearing the brunt of the Covid-19 pandemic, healthcare workers have significant risk of psychological distress and suicidal behaviour. Health-care worker suicide risk may be exacerbated by pre-existing or emergent mental health conditions, occupational stressors, organizational inadequacies, socio-economic and gender disparities. Nevertheless, resilience and post-traumatic growth may arise from increased global collaboration and collective altruism. Potential interventions in the health (physical \& psychological), as well as non-health spheres; and implications on suicide prevention in health-care workers will be discussed in the context of the Covid-19 pandemic. 